\title{
Dammar Resin Degradation Revisited
}

\author{
Andreas Buder*
}

\begin{abstract}
Dammar, a natural triterpenoid resin, is widely used as a picture varnish in art technology. Several research workers have recently characterised its composition and oxidative degradation. Due to a lack of sensitivity, conventional thermal methods to characterise oxidation stability (like TGA and DSC) often require a high temperature regime. Chemiluminescence measurements turned out to be more sensitive (temperatures below $100{ }^{\circ} \mathrm{C}$ ) and simultaneously allowed for better selectivity of the signal, which is directly coupled to the oxidation mechanism. The data collected here will enable an advanced kinetic modelling of Dammar degradation.
\end{abstract}

Keywords: Chemiluminescence · Dammar · Degradation · Oxidation · Varnish

\section{Introduction}

Varnishes made from triterpenoid natural resins have been used as surface coatings on paintings since the 17 th century. Despite typical chemical and physical degradation phenomena such as discolouration and hardening, dammar varnishes are still being applied in current restoration.

As a consequence of this limited lifetime, the removal of degraded dammar varnishes using organic solvents is still a standard measure in conservation-restoration. Side effects include partial removal of paint layer surfaces as well as leaching and swelling phenomena of paint structures due to the action of organic solvents.

Such interventions could be effectively reduced by applying more stable or stabilized varnish materials. While De la Rie and McGlinchey have researched possibilities of chemical stabilization of triterpenoid varnish materials, ${ }^{[1]}$ Dietemann focussed on selection criteria for Dammar resins. ${ }^{[2]}$

\section{The Origin and Composition of Dammar Resins}

Dammar resins can be harvested by tapping the bark of trees of the Dipterocarpaceae family which emcompasses over 500 species of tropical trees mainly from Southeast and Eastern Asia regions. The raw material is dried in open air. The dried lumps of resin are readily soluble in alcohol or in oil of turpentine (Fig. 1). On evaporation of the solvent, dammar forms a thin and transparent film with a smooth surface and of suitable hardness.

Despite their wide reaching botanical origin, Dammar resins have a fairly consistent composition with only minor variations of relative fractions. There has been considerable research on the composition and oxidation behaviour of triterpenoid resins. ${ }^{[2-5]}$ Chemically, triterpenoids form a large class of compounds having thirty carbon atoms, resulting biosynthetically from the cyclization of the unsaturated hydrocar-

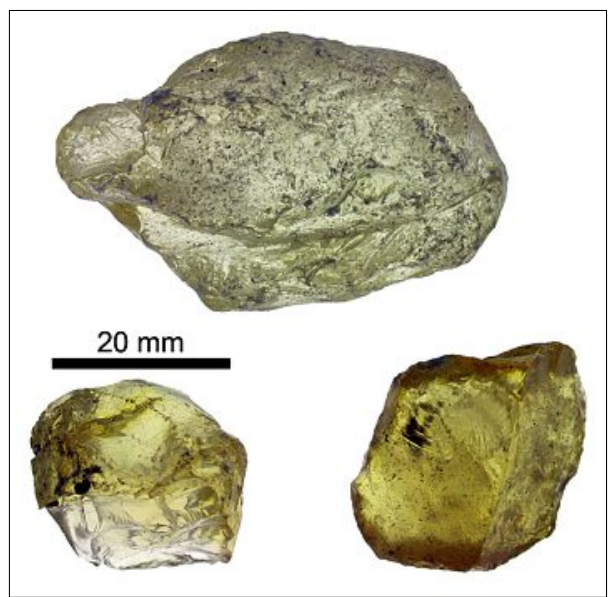

Fig. 1. Lumps of dammar resin raw material bon squalene. Watts and De la Rie found the following tetracyclic dammaranes to be major components: hydroxydammarenone (20-hydroxy-24-dammarene-3-one), dammarenolic acid (20-hydroxy-3,4-seco4(28),24-dammaradien-3-oic acid), dammaradienone (3-oxo-dammara-20(21),24diene) and dammaradienol (3ß-hydroxydammara-20,24-diene). Minor components are pentacyclic, oleanic derivates include ursonic acid (3-oxo-12-ursen-28-oic acid). ${ }^{6]}$ Other components are derivatives of the pentacyclic Hopane skeleton (Fig. 2). This is in good agreement with Mills and White. ${ }^{[3]}$ The functional groups determining the properties of triterpenoid molecules include hydroxyl, ketonic and carbon acid groups as well as double bonds.

\section{Ageing Process}

Dammar degradation results in severe discolouration and changes of mechanical properties. The yellowing and browning of Dammar films is accompanied by the formation of cracks and changes of surface properties. In general, degradation products seem to be more polar and less elastic than the original material. Recent analyses based on matrix-assisted laser desorption ionisation mass spectrometry (MALDI-MS), Fourier-transform infrared spectroscopy (FTIR) and gas chromatography-mass spectrometry (GC-MS) have largely contributed to the understanding of the dammar degradation processes. ${ }^{[2,4,7]}$ Important compounds like oleanonic acid (3-oxo-olean-12-en-28-oic acid) were identified as reference substances and alteration products thereof upon artificial ageing were carefully characterized..$^{[2,8]}$ 


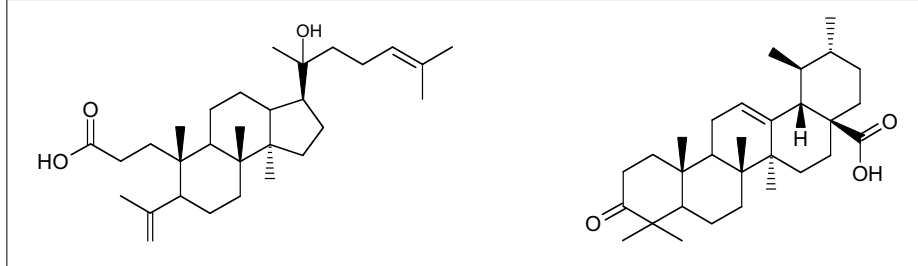

Fig. 2. Examples of triterpenoid structures: dammarenolic acid (left) and ursonic acid (right)

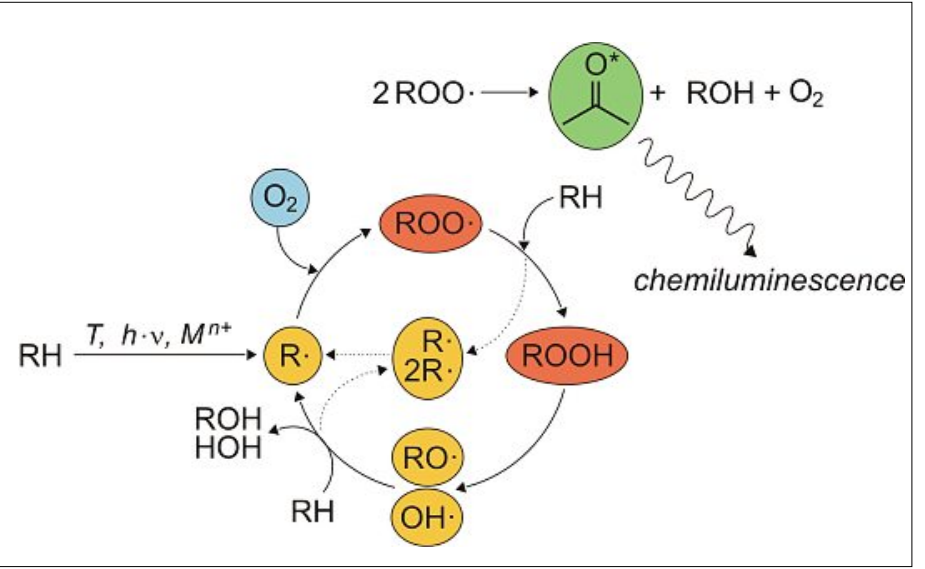

Fig. 3. Autooxidation cycle and the recombination process generating chemiluminescence (Russell mechanism)

Fragmentation and polymerisation of fragments are claimed to be responsible for the changes observed with Dammar resins: The corresponding reactions are induced thermally and under the influence of atmospheric oxygen and light. With respect to the original materials, oxidation products with smaller and larger masses can be detected in degraded dammar films, but the mean mass is lowered upon degradation. Dietemann ${ }^{22}$ explains this by a steady state equilibrium: the limited stability of oxidation- and polymerisation products leads to their decay. Fragments are then able to induce new polymerisation reactions. Zumbühl and coworkers suggests embrittlement of aged dammar films to be caused by increasingly polar and hence stronger intermolecular interactions of the oxidised fragments. ${ }^{[7.8]}$ This could also explain the phenomenon of blanching observed with aged dammar films to be a consequence of extreme swelling and crazing upon the action of water as a plasticiser. ${ }^{[9]}$

Dammar degradation follows a typical radical autooxidation mechanism (Fig. 3 ). While the influence of light is essential during initialisation, thermal propagation and termination processes occur in the absence of light. Using electron spin resonance spectroscopy (ESR), Dietemann ${ }^{[2]}$ has shown the radical content of freshly harvested triterpenoid resins to depend on the natural lighting during the drying process of the raw material. Daylight seems to induce the formation of semi-stable radical and oxidation products, which are the main cause for Dammar instability.

Starting radicals are formed photochemically by carbonyl absorption. This leads to bond fissure at the $\alpha$-carbon in a Norrish I type reaction. In triterpenoids this results in a ring cleavage and a double radical intermediate product (Scheme 1). Alternatively, a Norrish II type reaction leads to a non-radical cleavage product preserving the carbonyl function.

Oxygen readily reacts with the terpenoid skeleton alkyl radicals to form peroxyradicals. These are able to abstract hydrogen atoms from other skeletons. As a result, hydroperoxides and new alkyl radicals are formed. The $\mathrm{RO}-\mathrm{OH}$ bond is relatively weak and a homolytic bond cleavage can be induced thermally. The resulting alkoxy radicals can form new carbonyl or hydroxyl functional groups (Scheme 2), being a reason for the increased polar character observed in oxidised materials.

De la Rie and McGlinchey proposed conventional stabilisers to be used in dammar varnish film ${ }^{[1]}$ but their effectiveness has not yet been proven in kinetically controlled simulations.

\section{Experimental}

When compared to conventional thermo-analytical methods such as thermo- gravimetric analysis (TGA) or differential scanning calorimetry (DSC), chemiluminescence offers the advantage of higher sensitivity to oxidative reactions at very moderate temperatures. Furthermore, the intensity of the chemiluminescence signal is directly coupled to the autooxidative radical mechanism involved in the degradation of dammar: Chemiluminescence is produced in the visible region (380-450 $\mathrm{nm}$ ) by the relaxation of electronically exited carbonyl groups (Fig. 3). Käser et al. ${ }^{[9]}$ mention three different chemical mechanisms producing excited carbonyl groups in an auto-oxidation cycle.

The chemiluminescence apparatus used here has been developed by Käser et al. ${ }^{[9]} \mathrm{It}$ consists of a temperature-controlled ovenchamber with a sample stage coupled to a cooled photomultiplier tube (PMT) detector. The optical path between oven and PMT is equipped with a shutter unit, used to discriminate dark counts. Chemiluminescence signals can be optically filtered to select different spectral ranges. Oven chamber temperatures can be controlled by a computer to a precision of $+/-0.02 \mathrm{~K}$ in the range from $298 \mathrm{~K}$ to $450 \mathrm{~K}$. The oven chamber is continuously purged by a single gas or a mixture of gases (Fig. 4).

The chemiluminescence emission as a function of time is directly proportional to reaction rates of oxidation. The intensity of the chemiluminescence signal at a given time is a measure of the number of oxidation reactions occurring at that time.

Dammar samples (Kremer Pigmente GmbH \& Co. KG, DE-88317 Aichstetten, Dammar No. 60000, beste Qualität, handverlesen) were finely ground in an achate mortar and defined amount of some mil- 


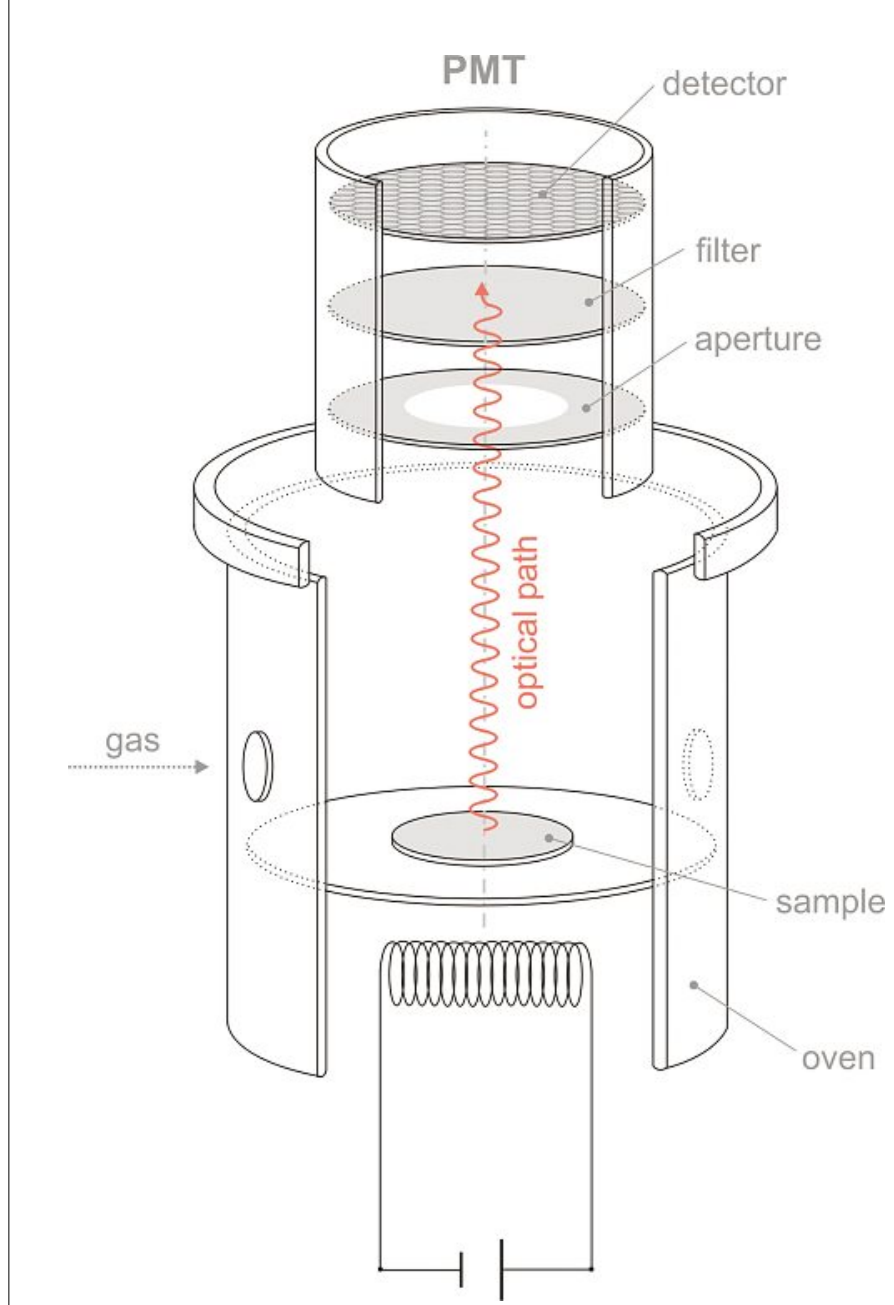

Fig. 4. Scheme of the chemiluminescence measuring device

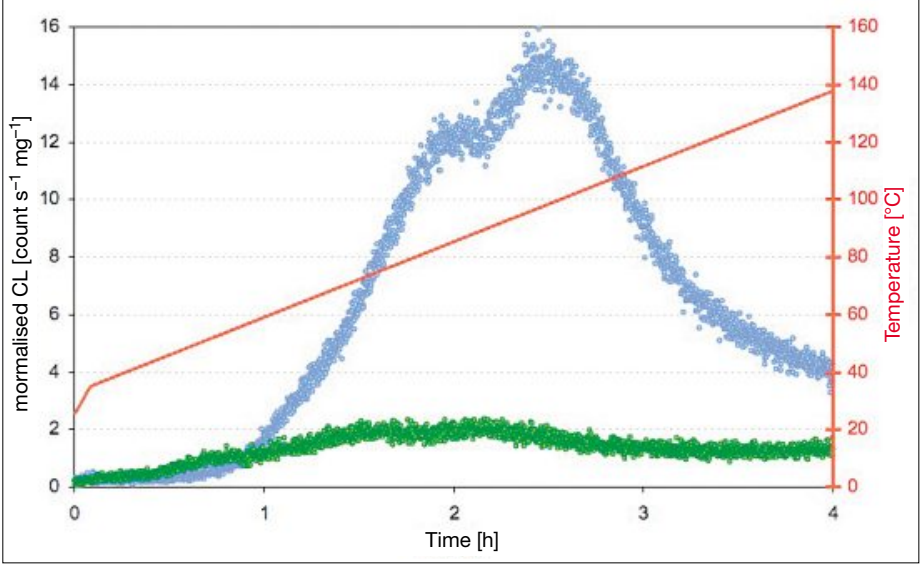

Fig. 5. Chemiluminescence signals of dammar samples taken from the surface (blue curve) and the inner (green curve) regions of a raw dammar lump. Measurement in pure nitrogen in the range from 308 to $413 \mathrm{~K}$ at a heating rate of $0.44 \mathrm{~K} / \mathrm{min}$.

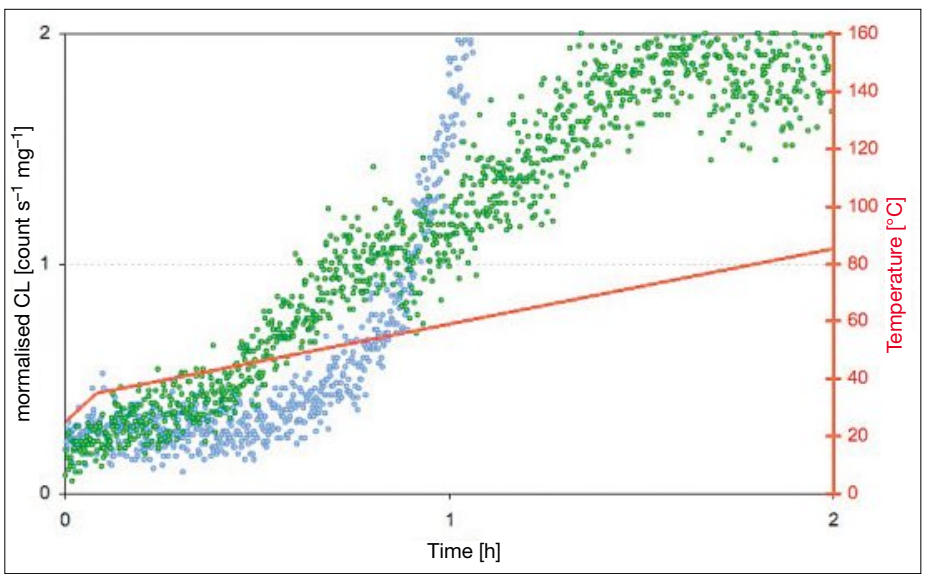

Fig. 6. Detail of Fig. 5: signal onset during the first $2 \mathrm{~h}$ ligrams directly placed into aluminium crucibles. All experiments were carried out in dynamic mode using a constant heating rate of $0.44 \mathrm{~K} / \mathrm{min}$ in the range from 308 $\mathrm{K}$ to $413 \mathrm{~K}$. A gas flowrate of $60 \mathrm{ml} / \mathrm{min}$ was chosen using either pure nitrogen and/ or pure oxygen. Chemiluminescence signals were normalized to one milligram of sample. All experiments were performed in triplicate.

Electron paramagnetic resonance (EPR) measurements were performed on a Bruker instrument, equipped with an electromagnet for fields up to 1.7 Tesla. Dammar samples were finely ground and a minimum of 100 milligrams was placed directly into a glass tube.

\section{Results and Discussion}

The first set of experiments compares samples taken from the surface region with samples from the core of a dried raw dammar lump. The corresponding signals were recorded in a pure nitrogen atmosphere (Figs 5 and 6).
As described by Dietemann, the surface regions of dammar lumps are pre-oxidised due to their exposition to light and oxygen during open-air drying in the harvesting process. Meta-stable oxidation products within the surface layer generally produce strong chemiluminescence signals with a maximum at $373 \mathrm{~K}$. After around $150 \mathrm{~min}$, the signal decreases due to the progressive consumption of oxidation products and the lack of oxygen in the atmosphere. The double maximum structure could be explained by a superimposed yellowing process of the sample, causing an increasing absorption of the blue chemiluminescence signal. A more probable explanation seems, however, that this structure is produced by the formation of transient oxidation products. This is supported by the fact that there is less signal produced by samples from the lump's core region. The onset of chemiluminescence in the low temperature region $(\mathrm{T}<330 \mathrm{~K})$ is more pronounced in samples taken from the inner, less oxidised regions. This could be interpreted as the presence of another kind of meta-stable radicals (possibly alkyl radicals) which already have reacted at the surface regions.
Another set of experiments was performed in a pure oxygen atmosphere. Obviously the samples from inner and surface regions can be very efficiently oxidized by the purging gas, thus producing a stronger signal than in nitrogen (Fig. 7). The signal onset shown in Fig. 8 is comparable but the samples from the inner regions seem to contain more oxidisable material. Again, semi-stable alkyl radicals could be responsible for the quick onset of oxidation and the multi-step oxidation processes in dammar being responsible for the curve's crossover after around $3 \mathrm{~h}$.

When comparing the signals from surface samples (Fig. 9) and samples taken from inner regions (Fig. 10) in nitrogen and oxygen, it becomes clear that in the outer regions there are more pre-oxidised materials and the presence of oxygen in the purging gas is not a prerequisite for an efficient oxidation onset. However, the raw material is still at the beginning of the oxidation process. For this reason, oxidation in the oxygen atmosphere is still much more effective than in nitrogen. With samples from inner regions, the delayed signal 


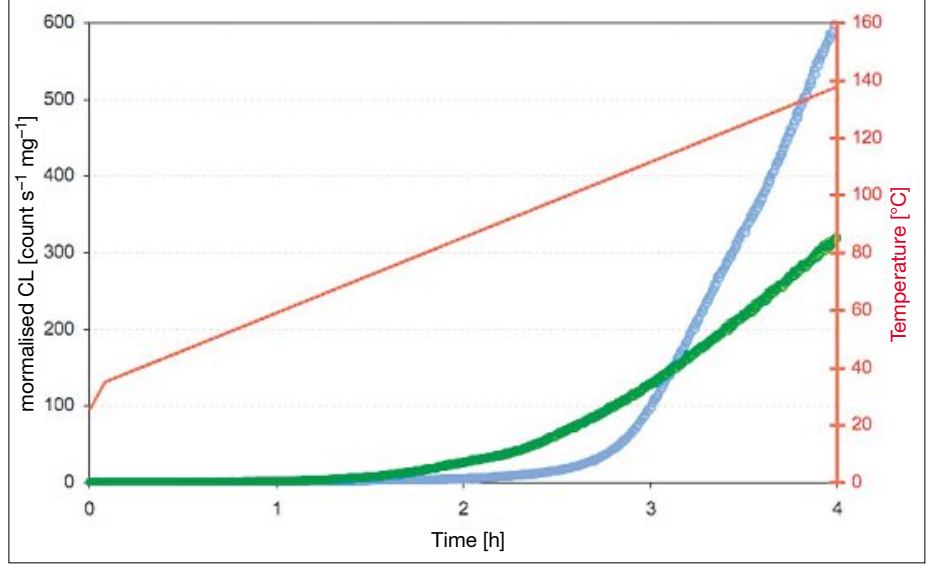

Fig. 7. Chemiluminescence signals of dammar samples taken from the surface (blue curve) and the inner (green curve) regions of a raw dammar lump. Measurement in pure oxygen in the range from 308 to $413 \mathrm{~K}$ at a heating rate of $0.44 \mathrm{~K} / \mathrm{min}$.

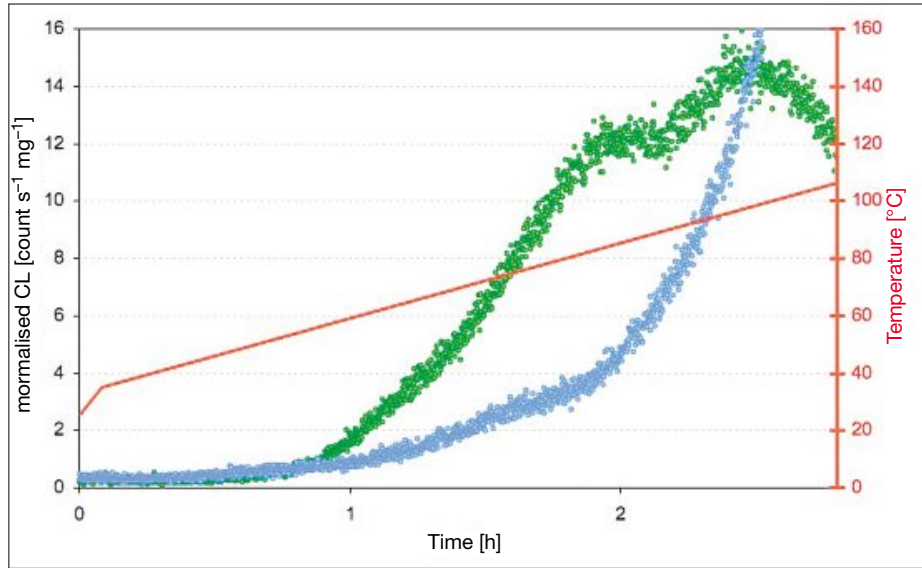

Fig. 9. Chemiluminescence signal onset $(2.8 \mathrm{~h})$ of dammar samples taken from the surface of a raw dammar lump. Measurements in pure oxygen (blue curve) and in pure nitrogen (green curve).

onset in oxygen (Fig. 10) is interpreted by the essential production of pre-oxidised material, leading to more efficient oxidation after around $1 \mathrm{~h}$. However, some preoxidized material must be present already from the beginning, as there is a detectable chemiluminescence signal decreasing after around $2 \mathrm{~h}$. The Table summarises the interpretation of the observations made on samples taken from core versus surface regions of dammar lumps.

The concentration of radicals at different depths of the dammar lumps has been analysed by EPR measurements (Fig. 11). Radicals are present throughout the lumps but their concentration is higher in the surface regions. This may be interpreted by different kinds of radicals being

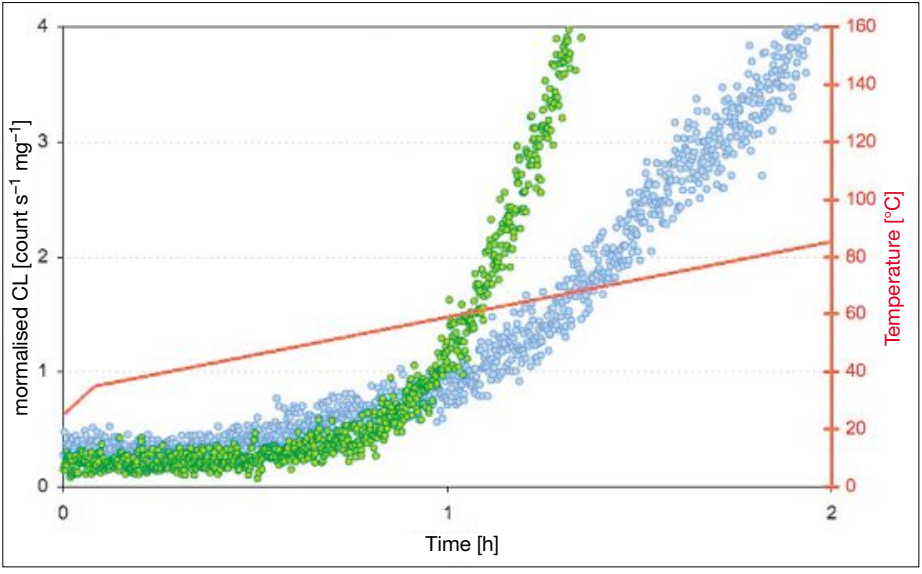

Fig. 8. Detail of Fig. 7: signal onset during the first $2 \mathrm{~h}$

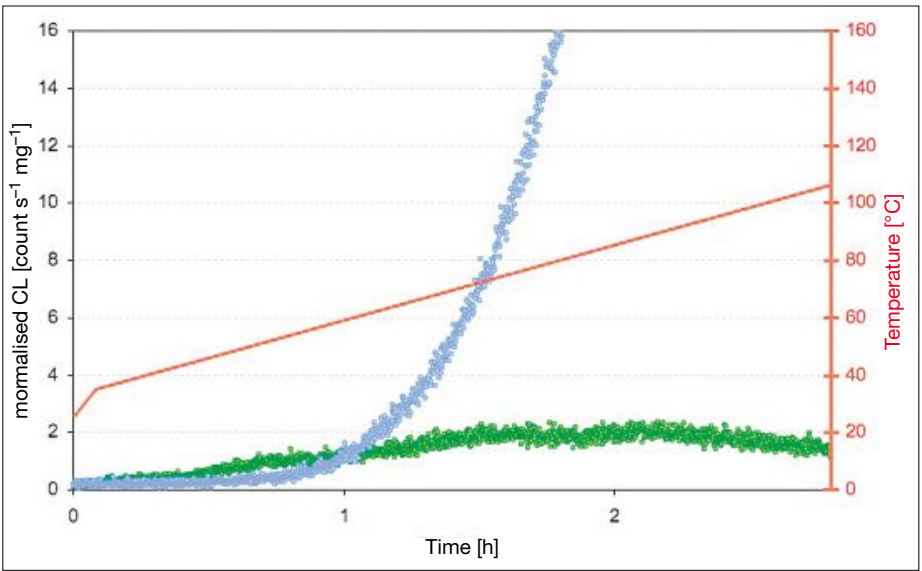

Fig. 10. Chemiluminescence signal onset $(2.8 \mathrm{~h})$ of dammar samples taken from the inner regions of a raw dammar lump. Measurements in pure oxygen (blue curve) and in pure nitrogen (green curve).

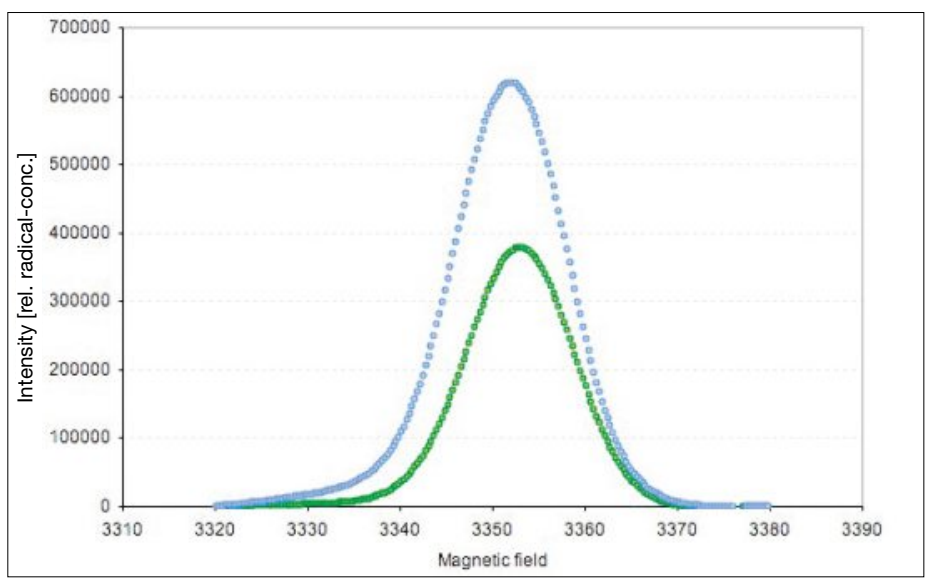

Fig. 11. Integrated EPR signals (relative intensities) of samples taken from the surface (blue line) and inner regions (green line) of raw dammar lumps

Table

\section{Samples from surface}

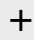

concentration of pre-oxidised material (including oxygen containing radicals)
Samples from core 
present at various levels of depth from the surface. Further EPR experiments will be performed to verify this hypothesis and to identify different radical species.

\section{Conclusions}

These preliminary results clearly show that chemiluminescence analysis is a suitable tool to characterize the oxidation behaviour of triterpenoid materials. This can be described as a typical multi-step chain reaction involving different activation energies. Isoconversional kinetic analysis will be applied to clarify this point. Based on the current findings, accelerated aging experiments, as widely applied in conservationrestoration research, turn out to be unsuitable for these materials. Lifetime prediction and evaluation of stabilization additives for triterpenoid resins should rather be based on advanced kinetic analysis. The quantification of the efficiency and effectiveness of stabilisation strategies for triterpenoid resins by chemiluminescence analysis could be a key to optimise their use in conservation-restoration practice.

\section{Acknowledgments}

The author would like to thank Dr. K.W. Kraemer, Department for Chemistry, Universität Bern for help with the EPR measurements and use of the EPR spectrometer. The author also thanks Fabian Käser (ACL Instruments) and Dr. Stefan Wülfert (Berner Fachhochschule,
Hochschule der Künste Bern) for discussion and collaboration. This project was funded by a research grant of the Berner Fachhochschule.

Received: October 13, 2008

[1] E. R. De la Rie, C. W. McGlinchey, Studies in Conservation 1989, 34, 137.

[2] P. Dietemann, 'Towards More Stable Natural Resin Varnishes for Paintings', Dissertationsschrift ETH No. 15224, ETH Zürich, 2003.

[3] J. S. Mills, R. White, 'The Organic Chemistry of Museum Objects', 2nd ed., Butterworth-Heinemann, London, 1994.

[4] G. Van der Doelen, 'Molecular studies of fresh and aged triterpenoid varnishes', PhD Thesis, University of Amsterdam, 1999.

[5] E. R. De la Rie, Studies in Conservation 1988, 33, 53.

[6] S. Watts, E. R. De la Rie, Studies in Conservation 2002, 47, 257.

[7] S. Zumbühl, R. Knochenmuss, S. Wuelfert, D. Frédéric, M. Dale, R. Zenobi, Analytical Chemistry 1998, 70, 715.

[8] S. Zumbühl, R. Knochenmuss, S. Wuelfert, Zeitschrift für Kunsttechnologie und Konservierung 1998, 12, 219.

[9] F. Kaeser, B. Roduit, S. Wuelfert, 'Safeguarded Cultural Heritage. Understanding \& Viability for the Enlarged Europe', Vol. 1, Proceedings of the 7th European Conference 'Sauveur', 31st May-3rd June 2006, Prague, Czech Republic, 2007, pp. 451-453. 